\title{
Identifying firing mammalian neurons in networks with high-resolution multi-transistor array (MTA)
}

\author{
A. Lambacher • V. Vitzthum • R. Zeitler • \\ M. Eickenscheidt • B. Eversmann • R. Thewes • \\ P. Fromherz
}

Received: 31 August 2010 / Accepted: 1 September 2010 / Published online: 16 September 2010

(c) The Author(s) 2010. This article is published with open access at Springerlink.com

\begin{abstract}
The electrical activity of a network of mammalian neurons is mapped with a Multi-Transistor Array (MTA) fabricated with extended CMOS technology. The spatial resolution is $7.4 \mu \mathrm{m}$ on an area of $1 \mathrm{~mm}^{2}$ at a sampling frequency of $6 \mathrm{kHz}$ for a complete readout of 16,384 sensor transistors. Action potentials give rise to extracellular voltages with amplitudes in a range of $500 \mu \mathrm{V}$. On the basis of the high resolution in space and time, correlation algorithms are used to identify single action potentials with amplitudes as low as about $200 \mu \mathrm{V}$, and to assign the signals to the activity of individual neurons even in a dense network.
\end{abstract}

\section{Introduction}

Cultured mammalian neurons are considered as model systems for certain aspects of brain functions such as the gating of ion channels, the response to drugs, the outgrowth of

A. Lambacher $\cdot$ V. Vitzthum $\cdot$ R. Zeitler $\cdot$ M. Eickenscheidt

P. Fromherz ( $\square)$

Department of Membrane and Neurophysics, Max Planck

Institute for Biochemistry, Martinsried, Munich, Germany

e-mail: fromherz@biochem.mpg.de

Fax: +49-89-8578-2822

B. Eversmann $\cdot$ R. Thewes

Corporate Research, Infineon Technologies, Munich, Germany

Present address:

B. Eversmann

Advanced Embedded Control, Texas Instruments Germany, Freising, Germany

Present address:

R. Thewes

Chair of Sensor and Actuator Systems, Faculty of Electrical Engineering \& Computer Science, Technical University Berlin, Berlin, Germany neurons, and the activity of neuronal networks. The investigation of network dynamics may provide a cue to elucidate the nature of the neuronal code and of neuronal memory. For that purpose, a large number and a high density of electrical sensors are required to record and to identify the activity of numerous individual neurons in a large network. Considering the size of mammalian neurons and the extension of neuronal networks, the spatial resolution must be at least $10 \mu \mathrm{m}$ on an area that is at least $1 \mathrm{~mm}^{2}$. To record extracellular voltages during an action potential (AP), the noise level must be below $100 \mu \mathrm{V}$ and the sampling frequency above $5 \mathrm{kHz}$. The sensor device should not affect the structure of the network. Thus a chemically homogeneous and biocompatible surface is required.

Since many years, passive metallic multi-electrode arrays (MEA) on various substrates are used to observe cultured mammalian neurons [1-7]. These devices fulfill the requirements of noise level and sampling frequency. However, the diameter of the electrodes is usually around $50 \mu \mathrm{m}$ and their spacing around $100 \mu \mathrm{m}$. The problem of sensor size and spacing was partially overcome with electrolyte-oxidesemiconductor field-effect transistors (EOSFETs) on silicon chips [8-12]. Another advantage of these devices was their chemically homogeneous surface that consisted of $\mathrm{SiO}_{2}$ silica, and later of $\mathrm{TiO}_{2}$, a most stable and biocompatible material $[13,14]$. The high spatial resolution, however, was attained only with linear EOSFET arrays because the interconnect lanes occupied most of the chip surface.

The problem of high resolution in two dimensions was solved with a multi-transistor array (MTA) that was fabricated by an extended complementary metal-oxidesemiconductor (CMOS) technology [15-17]. The array of $128 \times 128$ sensors had a pitch of $7.4 \mu \mathrm{m}$ with the interconnect lines buried in the chip. The chips were coated with a chemically homogeneous layer of $\mathrm{TiO}_{2} / \mathrm{ZrO}_{2}$. 


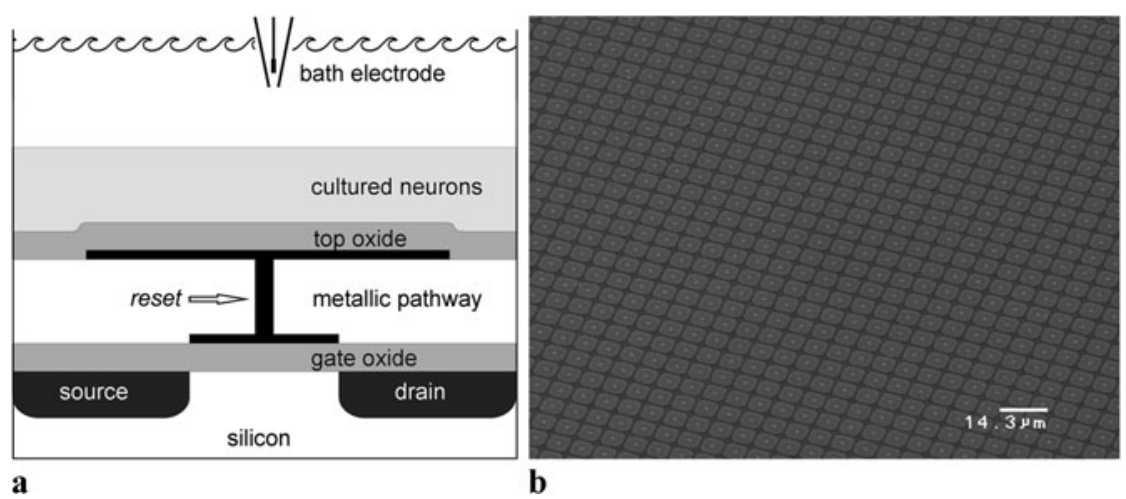

Fig. 1 Multi-Transistor Array (MTA) fabricated using an extended CMOS technology. (a) Schematic cross section of a sensor transistor with an electrolyte-oxide-metal-oxide-semiconductor (EOMOS) configuration (not to scale). The neurons are cultured on the top layer of titanium/zirconium dioxide that insulates a metallic pathway to the gate oxide of a standard metal-oxide-semiconductor field-effect transistor (MOSFET) with source and drain. Note that there is no floating gate node: the operating point of the MOSFET is adjusted by an auto-

The neurons were in contact with that oxide layer as with the EOSFET chips. That interface was joined to a common metal-oxide-semiconductor field-effect transistor. The resulting electrolyte-oxide-metal-oxide-semiconductor (EOMOS) configuration is illustrated in Fig. 1a. Undefined free-floating MOSFET gate nodes and fixed pattern noise due to process mismatch were overcome by an autozeroing circuitry in each pixel. This MTA device successfully yielded dynamic electrical maps on $1 \mathrm{~mm}^{2}$ of cultured snail neurons and of cultured brain slices $[16,17]$. A recording of cultured mammalian neurons was not possible with the first generation of MTA chips because the gate-referred RMS noise was too high due to the high bandwidth required for a complete sequential readout of the 16,384 sensors at a sampling frequency of $2 \mathrm{kHz}$ for the whole array. High frequency disturbances and noise, which are convoluted into the signal band, increased the noise to about $200 \mu \mathrm{V}$.

The crucial specification of the MTA chips with EOMOS configuration is a high spatial resolution with complete spatiotemporal mapping on the whole area and with a chemically homogeneous, biocompatible and insulating surface. The MTA chip differs from two kinds of active MEA chips. One development started with an array that had a spacing of $250 \mu \mathrm{m}$ with local filtering and amplification in each pixel of a CMOS chip [18]. In a more recent version, an array has been presented that has a pitch of $18 \mu \mathrm{m}$ of 11,016 metal electrodes where only 126 channels, however, can be read out simultaneously [19]. In another approach, an active MEA chip was described with a pitch of $42 \mu \mathrm{m}$ of 4096 metal electrodes on a CMOS chip with local filtering and amplification [20].

In the present paper we report on a significant progress of neuronal recording by MTA chips with EOMOS tran- zeroing circuit connected to the gate of the sensor MOSFET, indicated by the "reset" arrow in the figure. (b) Scanning electron micrograph. The surface of the chip is made of a chemically homogeneous and electrically insulating layer of $\mathrm{TiO}_{2} / \mathrm{ZrO}_{2}$. The metal contacts (platinum on tungsten pin) to the gates of the sensor transistors shine through the oxide layer. The total array consists of $128 \times 128$ sensor transistors on $1 \mathrm{~mm}^{2}$ with a pitch of $7.4 \mu \mathrm{m}$

sistors with respect to three aspects: (i) The RMS noise is lowered typically to $70 \mu \mathrm{V}$ and the sampling frequency for the whole array with 16,384 sensors is enhanced to $6 \mathrm{kHz}$. (ii) With these improvements it is possible to detect APs of mammalian neurons without signal averaging. (iii) Due to the high spatial resolution of AP recordings, the activity of individual neurons in a dense network can be identified by a correlation analysis.

\section{Materials and methods}

\subsection{Chip}

The entire CMOS chip has a die area of $5.4 \mathrm{~mm} \times 6.5 \mathrm{~mm}$. The pitch of the $128 \times 128$ sensor transistors is $7.4 \mu \mathrm{m}$ with an area $6.6 \mu \mathrm{m} \times 6.6 \mu \mathrm{m}$ of the sensor sites. Consequently, the total sensor area is $1 \mathrm{~mm}^{2}$. The chips have a chemically homogeneous and inert oxide surface made of $\mathrm{TiO}_{2} / \mathrm{ZrO}_{2}$. A scanning electron micrograph of the surface is shown in Fig. 1b. The surface profile is rather smooth with grooves of $40 \mathrm{~nm}$ depth and $800 \mathrm{~nm}$ width between the contact sites as probed by atomic force microscopy. The chips are wirebonded to a ceramic package. A perspex chamber is attached such that the sensor sites are exposed to the culture medium.

The mechanism of neuroelectronic recording by transistors in an EOMOS configuration is equivalent to the one of EOS transistors where the nature of interfacing has been studied in great detail $[10,11,21,22]$. In brief: the electrical excitation of a nerve cell gives rise to an extracellular voltage $V_{J}$ on the chip surface with respect to the electrical potential in the bath that is defined by a $\mathrm{Ag} / \mathrm{AgCl}$ reference 
electrode. In an EOMOS configuration, this voltage modulates the drain current through the serial capacitances of a top layer of $\mathrm{TiO}_{2} / \mathrm{ZrO}_{2}$ and the genuine gate oxide (Fig. 1a). A bias voltage is applied between chip and bath electrode, and operating points of the sensor transistors are adjusted in reset phases between measurements so that all sensor sites provide an identical electrical behavior. The dependence of the drain current on the voltage above the top oxide is determined through calibration by applying defined AC voltages to the bath electrode before neuronal recording.

Significant noise reduction compared to the first generation of the MTA chips [15-17] is achieved by redesigning the terminating and isolation circuitry for digital control signals, the reference generation and potentiostat, the power supplies, and the external amplifiers. Furthermore, a novel timing protocol for the readout allows an enhanced sampling rate for the full frame of 16,384 sensor transistors. As a result the gate-referred RMS noise is in a range of $40-80 \mu \mathrm{V}$, and the readout frequency for the whole array can be set to $6 \mathrm{kHz}$.

The relatively high level of noise arises from the requirement of a full mapping of the network activity by reading out all sensor transistors within each time frame. For that purpose, the 16,384 sensors are multiplexed through 16 analog output channels. In a first step, the signals of the 128 sensors of one line are multiplexed without local filtering through a line amplifier with an input bandwidth of $3 \mathrm{MHz}$. In a second step, the outputs of the 128 line amplifiers are again multiplexed to 16 analog output drivers of the chip. The total noise is dominated by the sensor transistors whereas amplification and multiplexing play a minor role. By aliasing, however, the noise of each sensor transistor in the bandwidth of $3 \mathrm{MHz}$ is projected into the final bandwidth of $3 \mathrm{kHz}$ when the full frame sampling rate is $6 \mathrm{kHz}$. As a consequence, the RMS noise is in a range of $40-80 \mu \mathrm{V}$, depending on variations of the chip fabrication process.

The noise for neuronal recording is determined by the noise of the detection system as well as by the JohnsonNyquist noise of the cell-transistor junction itself. The corner frequency of the Lorentzian power spectrum is around $60 \mathrm{kHz}$ for the junction of rat neurons [23], far smaller than the bandwidth of the chip itself. Thus by aliasing, the total thermal noise of the junction is projected into the final bandwidth of $3 \mathrm{kHz}$. The integral contribution to the RMS noise is $\sqrt{k_{B} T / C_{\text {sens }}}$ with the thermal energy $k_{B} T$ and the capacitance $C_{\text {sens }}$ of the sensor-cell contact. The resistance $R_{J}$ of the cell-chip junction does not appear in that relation. With an area specific capacitance around $1 \mu \mathrm{F} / \mathrm{cm}^{2}$ and a contact area around $40 \mu \mathrm{m}^{2}$, the RMS noise is on the order of $100 \mu \mathrm{V}$ for a fully covered sensor electrode.

\subsection{Cell culture}

Nerve cells from the rat hippocampus were cultured according to standard procedures [24]. Hippocampi from E19 rats (Wistar outbred) were harvested and digested for $20 \mathrm{~min}$ at $37^{\circ} \mathrm{C}$ in phosphate buffered saline (PBS, Gibco Invitrogen, 14040-091) containing 0.5\% bovine serum albumin (BSA, Sigma-Aldrich, A-8806, fraction V powder, fattyacid free, low endotoxin) and $0.5 \mathrm{mg} / \mathrm{ml}$ papain (SigmaAldrich, P4762). Excess medium was removed and cells resuspended in $2.5 \mathrm{ml}$ Dulbecco modified Eagles's medium (DMEM, Gibco Invitrogen, 61965-059) with 10\% fetal bovine serum (FBS, Gibco Invitrogen, 10270-098, batch $40 \mathrm{~F} 6414 \mathrm{~K}$ ). After centrifugation at $1200 \mathrm{rpm}$ for $10 \mathrm{~min}$, excess medium was removed and the cells were pre-plated for $60 \mathrm{~min}$ in $10 \mathrm{ml}$ DMEM on a $10 \mathrm{~cm}$-diameter NUNC plate with Nucleon surface (Nalge Nunc International, 150350). After a second pre-plating for $30 \mathrm{~min}$, the cells were centrifuged and re-suspended in $1 \mathrm{ml}$ DMEM. The cell density was adjusted with DMEM to 300,000 per $\mathrm{mL}$.

CMOS chips with culture chamber attached were cleaned three times by cautious wiping with oil-free Q-Tips with a slightly basic detergent at $80^{\circ} \mathrm{C}(5 \%$ Tickopur R36, Dr. H. Stamm GmbH, Berlin). Further sterilization was accomplished by irradiation with UV light for one hour. The surface was functionalized by coating overnight with polyL-lysine (molecular weight 150,000 to 300,000 , SigmaAldrich P 1274) from a $0.1 \mathrm{mg} / \mathrm{ml}$ solution in aqua ad (Braun, Melsungen). A volume of $0.5 \mathrm{ml}$ of the cell suspension was applied and supplemented with $1 \mathrm{ml}$ of Leibovitz15 (Gibco Invitrogen, 31415-086) with 5\% FBS. After incubation for $1 \mathrm{~h}$, the medium was changed to $1.5 \mathrm{ml}$ neurobasal medium (NB, Gibco Invitrogen, 21103-049) with B27 supplement (Gibco Invitrogen, 17504-044) and glutamax I (Gibco Invitrogen, 35050-038). The prepared CMOS chips were kept in an incubator at $37^{\circ} \mathrm{C}$ with $10 \% \mathrm{CO}_{2}$ for three weeks. Once a week, $200 \mu \mathrm{l}$ of medium was removed and $400 \mu \mathrm{l} \mathrm{NB}$ medium was added.

\subsection{Electron micrographs}

CMOS chips without attached chamber were placed in a Petri dish and neurons were cultured for three weeks as described above. The fixation procedure followed Ref. [25]. In a first step of fixation, the culture medium was removed and a glutardialdehyde solution $(2.5 \%)$ in $150 \mathrm{mM}$ sodium cacodylate buffer, $\mathrm{pH} 7$, was added for $10 \mathrm{~min}$. That procedure was applied six times. After $1 \mathrm{~h}$, the chips were rinsed three times with cacodylate buffer and kept in a refrigerator for one day. In a second step of fixation, the buffer was removed, and a $1 \%$ solution of $\mathrm{OsO}_{4}$ in $100 \mathrm{mM}$ cacodylate was applied for a few minutes. The procedure was repeated. Finally the chip was rinsed three times with pure water. In 
a first step of dehydration, the water was sequentially replaced by 25, 50, 75 and $100 \%$ ethanol, waiting for $15 \mathrm{~min}$ after each exchange. In a second step of dehydration in a glass beaker, the ethanol was replaced by a 1:1 mixture of ethanol/acetone and by pure acetone. For drying, the chips were transferred into a bowl with acetone and placed into a critical point dryer. The acetone was replaced by liquid carbon dioxide. Above its critical point, the carbon dioxide was removed. The dry chips were attached to the metal holder of the electron microscope using a conductive adhesive. After drying for one day, gold was sputtered with an average thickness of $6 \mathrm{~nm}$. The electron micrographs were taken at $10-20 \mathrm{keV}$.

\subsection{Protocol}

The cultivated CMOS chips were mounted in the setup where the temperature was held at $37^{\circ} \mathrm{C}$ with a Peltier element using an integrated temperature sensor on the chip. The measurements were performed with the neurobasal culture medium. At first, the sensor transistors were calibrated by applying a sine voltage with a peak-to-peak amplitude of $3 \mathrm{mV}$ to the $\mathrm{Ag} / \mathrm{AgCl}$ electrode. Then the spontaneous activity of the neurons was observed for periods of $600 \mathrm{~ms}$ with intervals of several seconds. This restriction was due to the fact that the current setup does not allow for continuous streaming of measured data to storage devices. In these intervals, the reset protocol for autocalibration was performed by adjusting the operating points of the sensor transistors. To enhance the frame rate to $24 \mathrm{kHz}$ the array was divided into four stripes (128 lines, 32 columns) that were measured sequentially. To correct the data offline for a slight drift, a baseline was determined for each sensor transistor by applying a floating time average that was subtracted from the raw data. A window of 151 data points, corresponding to duration of $6.3 \mathrm{~ms}$, was chosen such that the shape of recorded APs was not significantly altered.

\subsection{Data evaluation}

The mapping of neuronal activity at a high spatial resolution with the CMOS chip gives rise to several problems of data evaluation. (i) First, the full mapping by the chip results in relatively high noise. (ii) Further, the signal of a single cell is usually visible on several sensor transistors. Care must be taken that all recorded signals are correctly assigned to the corresponding AP. (iii) On the other hand, signals of several cells may be recorded by only one sensor transistor. Therefore each detected AP must be assigned to the proper cell.

Traditionally, AP recordings of extracellular electrodes are evaluated with a threshold detector. Spike sorting is usually based on principal component analysis (PCA) [26]. For evaluation of data measured with high density MEAs, where individual neurons are detected on different electrodes, template matching and extended PCA algorithms were used $[27,28]$. With the current CMOS chip, we can detect action potentials with high confidence at a supreme spatial resolution of $7.4 \mu \mathrm{m}$, even if the signal-to-noise ratio is as low as 3 . This spatial resolution of the chip facilitates spike sorting through a correlation analysis of signals on several sensors below one cell. We proceed in three steps: the selection of significant signals, the identification of action potentials (APs), and the assignment of APs to individual neurons.

Significant data points To identify data points that significantly deviate from the noisy baseline, no simple threshold approach can be applied. The whole chip generates $10^{8}$ data points per second. If we allow for one false positive signal per second, we need to make the probability of such an event $X$ as low as $10^{-8}$. The threshold $V_{0}$ for a Gaussian noise with variance $\sigma^{2}$ is given in (1) where $V$ is the measured extracellular voltage.

$$
\begin{aligned}
P(X) & =P\left(V>V_{0}\right) \\
& =\int_{V_{0}}^{\infty} d v \frac{1}{\sqrt{2 \pi \sigma^{2}}} \exp \left(-\frac{v^{2}}{2 \sigma^{2}}\right)=10^{-8}
\end{aligned}
$$

Solving (1) numerically yields $V_{0}=8.2 \sigma$. For $\sigma=$ $70 \mu \mathrm{V}$, the threshold is $V_{0}=570 \mu \mathrm{V}$ and many APs would not be detected. To overcome the problem, we take advantage of the temporal and spatial resolution of the chip. Since the sampling time is shorter than the duration of an AP and the pitch smaller than the size of a neuron, the same AP is detected in several consecutive time frames and at several adjacent sites. For each time frame at each site, we consider the joint probability that the signals $V_{i}$ in a spatial $3 \times 3$ neighborhood in 3 consecutive time frames significantly deviate from the baseline, i.e. the length $|\mathbf{V}|$ of the 27-dimensional signal vector constructed from the 27 data points must exceed a threshold $V_{0}$. The threshold is defined by the lower bound of (2) for a 27-dimensional ellipsoid with $\sum_{i=1}^{27} V_{i}^{2} / \sigma_{i}^{2}=$ const.

$$
\begin{aligned}
P(X)= & P\left(|\mathbf{V}|>V_{0}\right) \\
= & \int_{V_{0,1}}^{\infty} \cdots \int_{V_{0,27}}^{\infty} d v_{1} \cdots d v_{27} \\
& \times \prod_{i=1}^{27} \frac{1}{\sqrt{2 \pi \sigma_{i}^{2}}} \exp \left(-\frac{v_{i}^{2}}{2 \sigma_{i}^{2}}\right)=10^{-8}
\end{aligned}
$$

By numerical integration-upon a substitution $V_{i}^{\prime}=$ $V_{i} / \sigma_{i}$ and a lower bound of a 27-dimensional sphere-we obtain the threshold condition $\sum_{i=1}^{27} V_{i}^{2} / \sigma_{i}^{2}=(9.5)^{2}$. If a neuron was coupled equally to the 9 sensor transistors with the same $\sigma_{i}=70 \mu \mathrm{V}$, an AP would be detected for an extracellular voltage of $V_{i}=127 \mu \mathrm{V}$. 
A certain problem is that the noise of the real transistor recordings is not always Gaussian, but occasionally shows distinct tails. We attribute this effect to digital crosstalk on the chip and to the fact that the noise is not constant during a measurement of $600 \mathrm{~ms}$. Finally, the threshold was empirically set to $(11.7)^{2}$ and sensors with extremely strong variations of the noise were eliminated.

Action potentials Action potentials (AP) in neurons give rise to transients of the extracellular voltage on a sensor transistor. These transients can have different waveforms, depending on the distribution of ion channels in the cell membrane and on the position of the sensor transistor relative to the cell $[21,22]$. The problem of the second step of evaluation is to collect all significant data points that belong to a single AP. This set of signals on different sensor sites and time frames has the shape of a spindle in the space-time. The spindle may have gaps in space and time due to noise, to inhomogeneous cell-chip coupling in space, or to a biphasic waveform in time. The main problem here is to prevent an AP from being split into several ones due to the gaps. At first we assemble significant signals that occur on adjacent sites in a chosen time frame into a signal cluster. Due to gaps there may exist more than one cluster for one AP in a single time frame. Then these signal clusters in different time frames are combined if they overlap in space at least with one other cluster. In that search, a window of 10 time frames is chosen such that short gaps without significant signals are bridged, and that consecutive APs are not merged. The transistor and the time frame which are closest to the center of gravity of all signals in a spindle are chosen as a representative space-time event of an AP.

Neurons In a third step of evaluation, we have to assign the AP recordings to individual nerve cells. If nerve cells grow in close neighborhood, their range of cell-chip coupling may overlap. A certain transistor may record APs from different cells. For all APs that share a sensor we have to decide whether they belong to the same cell or to different cells. The analysis relies on the similarity of recorded signals from different APs that originate from the same cell. Therefore the coefficients $c_{i j}$ of cross-correlation for pairs of spindles $i$ and $j$ are normalized according to (3), where $k$ runs over a certain subset (see below) of signals of the spindle.

$c_{i j}=\sum_{k} V_{i, k} V_{j, k} / \sqrt{\sum_{k} V_{i, k}^{2} \sum_{k} V_{j, k}^{2}}$

We proceed in two steps. In the first step we look for "related spindles" that eventually belong to APs in the same cell. Here it is important to collect all spindles of a certain cell. If a spindle of a certain cell is not properly assigned to the related cell, a surplus cell is identified. In the second step we examine whether such a set of related spindles must be partitioned into spindles that belong to APs in different cells.

Two spindles $i$ and $j$ are considered to be related if their correlation coefficient $c_{i j}$ is positive with respect to a limited subset of signals $k$. We align the representative events of two spindles in time and define cores of $3 \times 3$ sites around the representative events. The correlation coefficients are evaluated only from significant signals from sensors which belong to the cores of aligned spindles in time. For a positive correlation, the cores of two spindles must overlap and the variation of the signals in time must have a certain similarity.

This is due to the fact that the coupling area of a cell, i.e. the area over which a signal can be detected, often is larger than the physical dimensions of a neuron. We assume that neurons and/or glial cells can form tight clusters where the extracellular space below the cells is well insulated from the bath and therefore the electrical length constant is larger than the diameter of a cell. We also assume that the largest extracellular signal of a cell is detected at the same position for different APs. So if two spindles belong to one cell, we assume that their cores should overlap spatially. Spindles generated by one cell have similar shapes in space-time. We define sets of related spindles, where each spindle is positively correlated at least with one other spindle in the set.

In the second step, we examine whether a set of related spindles must be split into subsets that belong to different cells. Again we evaluate the coefficients of cross-correlation between pairs of spindles, now with a different choice of signals $k$. We choose all sites that give rise to significant signals in a set of related spindles. Thus data points from the outer rim of the spindles contribute where the data still shows the AP, but where the neighborhood prevented it being over the threshold when finding significant signals. As a consequence the cross-correlation becomes more selective. The symmetric matrix of the correlation coefficients is sorted. We start with a certain row $i$ that refers to the correlation of a spindle with all other spindles $m$ and look for a row $j$ of another spindle with a most similar correlation by computing the sum of squared differences $\sum_{m}\left(c_{i m}-c_{j m}\right)^{2}$. We place the row $j$ with the lowest value next to the row $i$. This procedure is iterated for a complete cycle of rows and repeated for all rows used as starting row. The matrix with the lowest overall sum of squared differences of correlation coefficients is taken as a sorted matrix. It shows a distinct structure if the spindles in the related set are from more than one neuron. All connected sets of spindles are evaluated and split if necessary. The correct splitting is confirmed by eye inspection of the individual spindles. 


\section{Results and discussion}

Nerve cells from the rat hippocampus were cultured in serum-free medium for three weeks on the EOMOS array coated with poly-L-lysine. The conditions were similar to those in previous experiments with EOS transistors [11, 12] and in various experiments with MEAs [1-7].

\subsection{Rat neurons on EOMOS chip}

Several EOMOS chips with cultured neurons were investigated by scanning electron microscopy. An area of about $500 \mu \mathrm{m} \times 500 \mu \mathrm{m}$ is depicted in Fig. 2a. It shows about 300 neuronal cell bodies and a dense web of neurites that covers the chip surface. Figure $2 b$ illustrates the size and pitch of the sensor transistors with the dimension of the nerve cells. Figure $2 \mathrm{c}$ shows how the cell bodies are embedded at various depths in the web of the neurites with a width of about $0.3 \mu \mathrm{m}$. Some neurites form plate-like structures as depicted in Fig. 2d.

The electron micrographs closely resemble those of classical experiments with cultured rat neurons [25]. They demonstrate two important issues: (i) There is no visible effect of the chip structure on the outgrowth of the neurons.
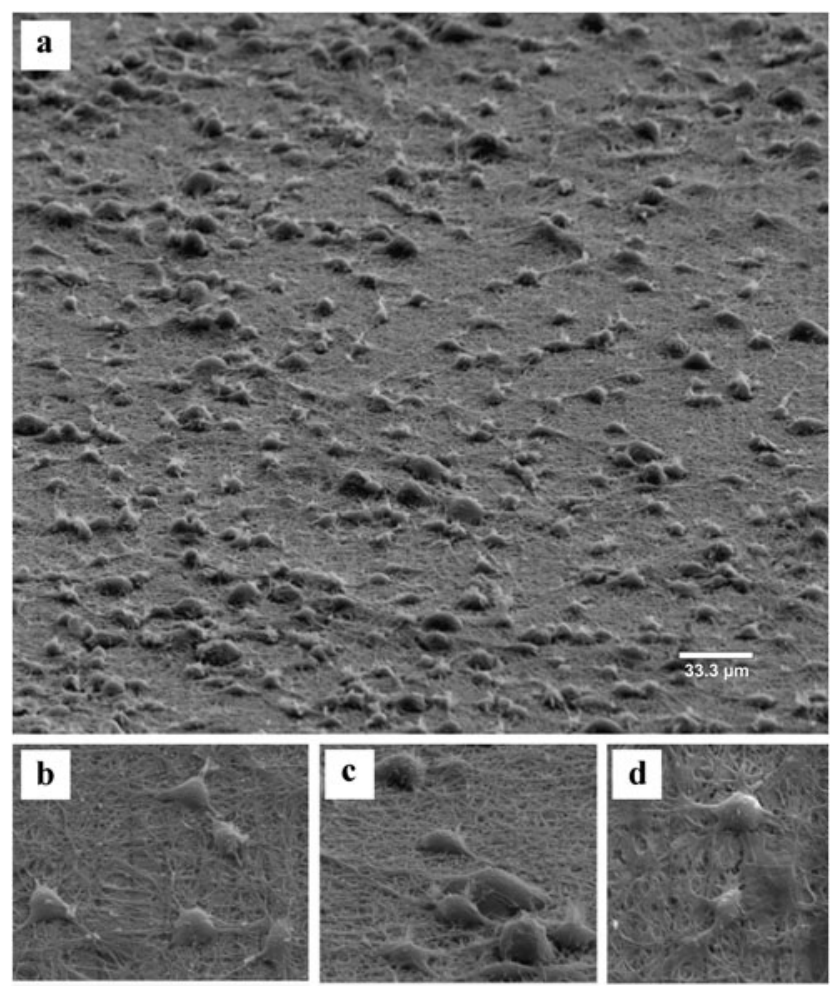

Fig. 2 Multi-Transistor Array (MTA) with cultured rat neurons. Scanning electron micrographs with cells that were cultured for three weeks in serum-free medium on the $\mathrm{TiO}_{2} / \mathrm{ZrO}_{2}$ surface of the chips coated with poly-L-lysine. The scale is given by the pitch of $7.4 \mu \mathrm{m}$ of the array that shines through the web of neurites
This feature is due to the homogeneous chemical nature and the modest topographical profile of the chips surface. (ii) Each neuron covers one or several transistors due to the small size of the sensors and their high density.

The electron micrographs give rise to several questions about the electrical interfacing of neurons and transistors: Are the cell bodies in tight contact with the oxide surface of the sensor transistors or are they separated by the dense web of neurites? Are there tight contacts formed only by the cell bodies or also between arborized neurites or plate-like neurites far from the cell bodies? Does the dense web of neurites form a layer of enhanced resistivity that is able to seal cell-chip junctions?

\subsection{Extracellular voltage}

We recorded the spontaneous activity of cultured neuronal networks. The response was calibrated in terms of the average extracellular voltage at the oxide surface of the EOMOS transistors $(6.6 \mu \mathrm{m} \times 6.6 \mu \mathrm{m})$ with respect to the bulk electrolyte. Various waveforms of the voltage transients caused by APs were observed. Four representative examples with large amplitudes are depicted in Fig. 3. They were recorded at a sampling frequency of $24 \mathrm{kHz}$ without temporal or spatial filtering. (The quality of the recordings was almost unchanged if a sampling frequency of $6 \mathrm{kHz}$ was used.) The prevalent waveform $(80 \%)$ was a negative transient (trace III) with a width at half maximum of about $1 \mathrm{~ms}$. Some of the negative peaks were very sharp (trace IV). In addition, biphasic waveforms with a primary positive peak were observed (trace II). Occasionally, purely positive transients were found (trace I). The relatively high noise of the recordings is due to the contribution of the thermal noise in the neuron-chip junction as described in the Materials and Methods section.

The extracellular voltage $V_{J}(t)$ in the junction of cell and chip depends on the intracellular voltage $V_{M}(t)$ during an

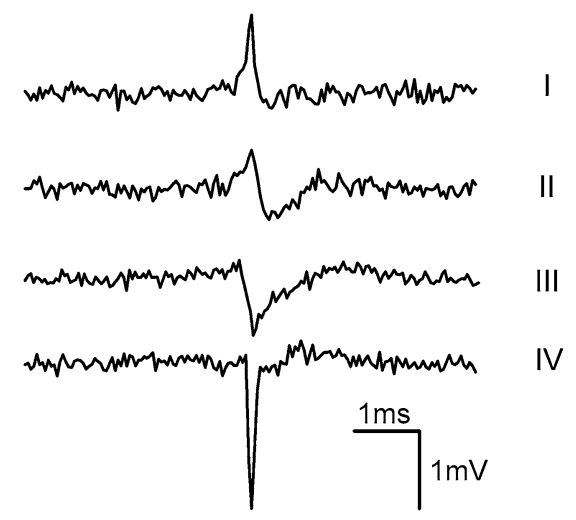

Fig. 3 Typical waveforms of the extracellular voltage recorded by sensor transistors, raw data with a sampling frequency of $24 \mathrm{kHz}$. The dominating waveform is trace III 
Fig. 4 Recordings of action potentials and noise. Top: typical recording of a sensor transistor underneath an active neuron. Data points that belong to action potentials are marked with red arrows. The signal at $216 \mathrm{~ms}$ is smaller than numerous peaks due to noise. A simple threshold detector would not identify this action potential. Bottom: the blow-up shows the signal at $216 \mathrm{~ms}$ together with the time traces of the eight surrounding sensors. The extracellular signal appears not only on the central sensor, but also on adjacent sensors. This correlation is used to identify the action potential

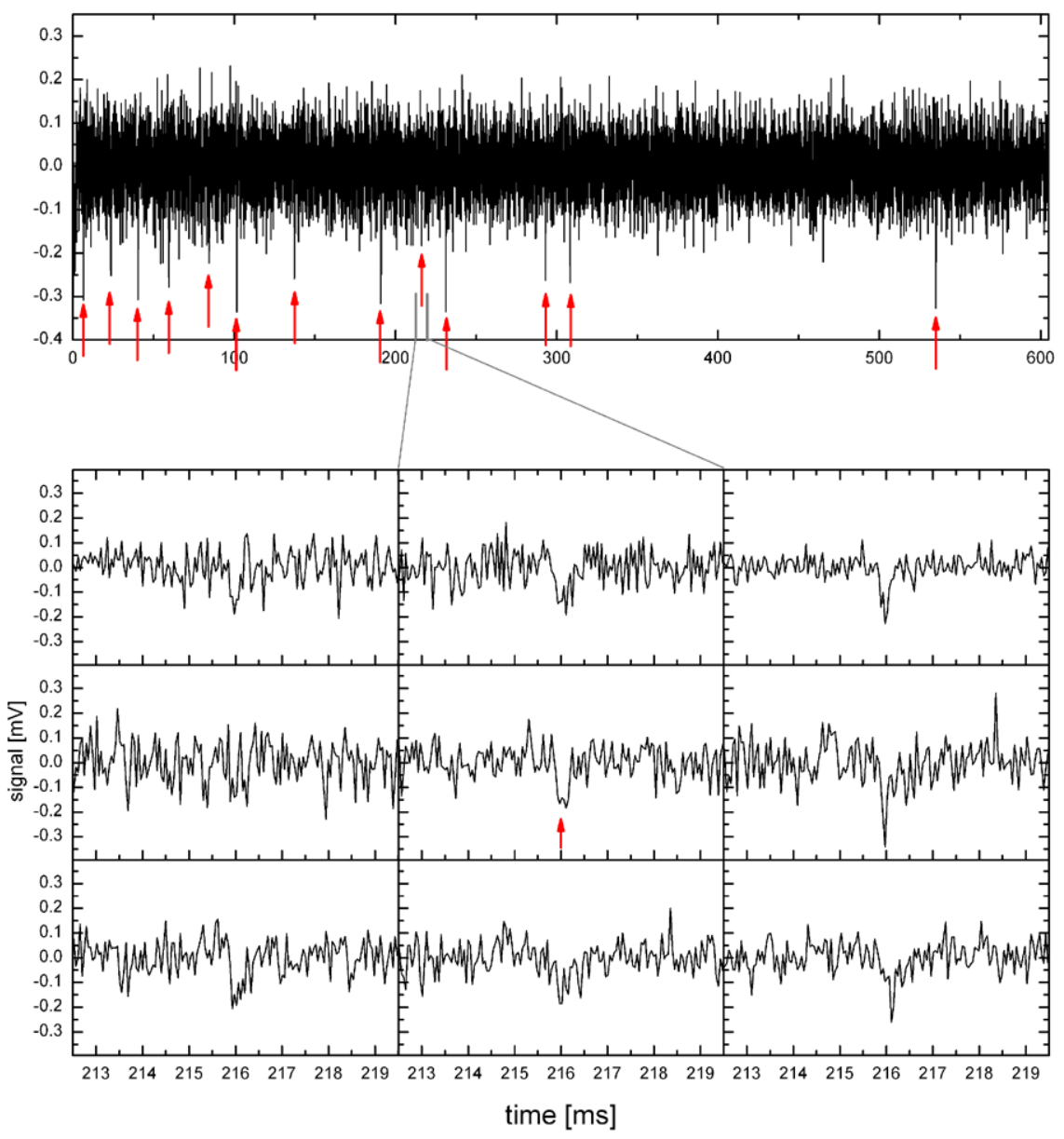

AP. It is determined by the ionic and capacitive currents that flow through the adherent membrane and give rise to a voltage-drop along the resistance of the cell-chip junction $[21,22]$. When the capacitive current is expressed by the ionic current through the total cell membrane, the extracellular voltage $V_{J}(t)$ can be described by (4) if the signals are small [22].

$V_{J} \approx g_{J}^{-1} \sum_{i}\left(g_{J M}^{i}-g_{F M}^{i}\right)\left(V_{M}-V_{0}^{i}\right)$

Here, $g_{J M}^{i}$ and $g_{F M}^{i}$ are the area-specific ion conductances of the adherent and free membrane with reversal voltages $V_{0}^{i}$. The parameter $g_{J}=\eta_{J} / r_{J} A_{J}$ is an areaspecific conductance of the cell-chip junction where $r_{J}$ and $A_{J}$ are the sheet resistance and the area of the junction. $\eta_{J}$ accounts for the position of the transistor with $\eta_{J}=4 \pi$ for a recording in the center of a circular junction [21]. The waveform $V_{J}(t)$ depends on inhomogeneous ion conductances $g_{J M}^{i} \neq g_{F M}^{i}$ during an AP. The different recordings of Fig. 3 indicate that transistors probe neuronal activity in different functional parts of the neurons. The dominating negative transients are due to an inward sodium current in junctions with $V_{M}-V_{0}^{i}<0$ and $g_{J M}^{N a}>$ $g_{F M} \mathrm{Na}$. That assignment implies that the most efficient interfacing takes place at the spike-generating regions of the cells.

\subsection{Identification of action potentials}

The amplitude of extracellular recordings depends on the quality of the cell-transistor junctions according to (4). In order to probe a neuronal network, it is essential to obtain AP recordings from as many cells as possible on a chip at the given noise level. We proceed in two steps.

First, we identify data points that significantly deviate from the baseline, by taking advantage of the high temporal and spatial resolution of the chip. Figure 4a shows the typical recording of a sensor transistor. Data points that finally are assigned to an action potential are marked by red arrows. Some of them are smaller than noise peaks. E.g., the arrow at time 216 ms marks a data point that by itself is not significant. Such a data point, however, can be identified to be significant if the correlation is taken into account with adjacent data points in space and time. The issue is 


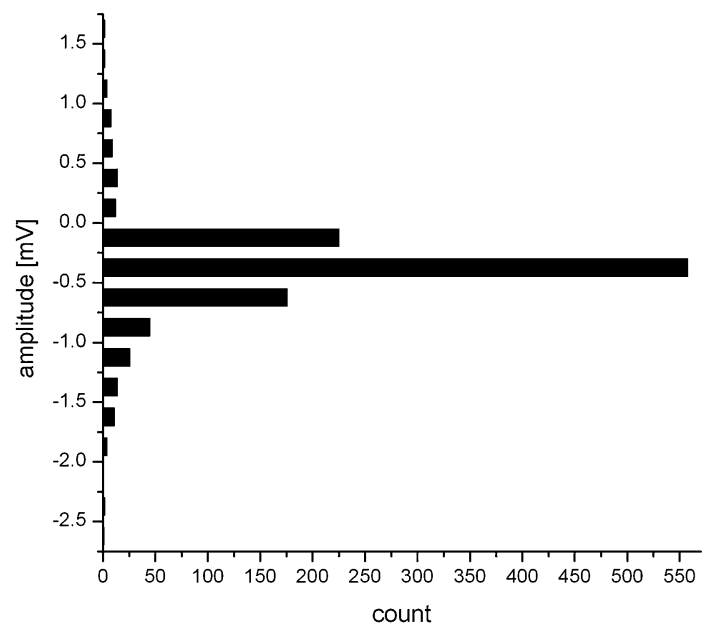

Fig. 5 Histogram of the extracellular voltage amplitudes of action potentials from cultured rat neurons as measured with Multi-Transistor Array

illustrated in Fig. 4b that shows the time traces of nine adjacent recording sites. The signal marked with an arrow at the central sensor pixel is also visible on several neighboring sensors. Details are described in the Materials and Methods section.

As a second step, we collect the significant data points that belong to the same AP. In principle, such a set of signals forms a spindle-shaped volume in space-time. In reality such a spindle is fragmented due to noise, to a biphasic waveform and to inhomogeneous cell adhesion. To identify a spindle that represents an AP, an algorithm is used that takes into account certain properties of APs as described in the Materials and Methods section. The center of gravity of a spindle defines the space-time event of an AP.

From these identified APs we can gather the amplitudes of the extracellular signals shown as a histogram in Fig. 5. At an RMS noise of typically $70 \mu \mathrm{V}$, the threshold of recording is about $200 \mu \mathrm{V}$. There is a wide distribution of amplitudes due to a different quality of the cellchip junctions with a median around $0.5 \mathrm{mV}$. The result confirms previous experiments with simple EOS transistors $[11,12]$. The signals are rather high in comparison with most recordings by planar metal electrodes (MEA). It may be emphasized that all transistor recordings represent true extracellular voltages due to the calibration protocol before each measurement. This identification has been verified with micropipette recordings in acute brain slices on transistor chips [29].

The distribution of AP recordings in space is shown in Fig. 6. The data are recorded at $24 \mathrm{kHz}$ for 10 periods of $600 \mathrm{~ms}$ for each of four vertical stripes of transistors as indicated in the figure. The recordings exhibit clusters of recording sensor transistors. A single cluster, however, can-

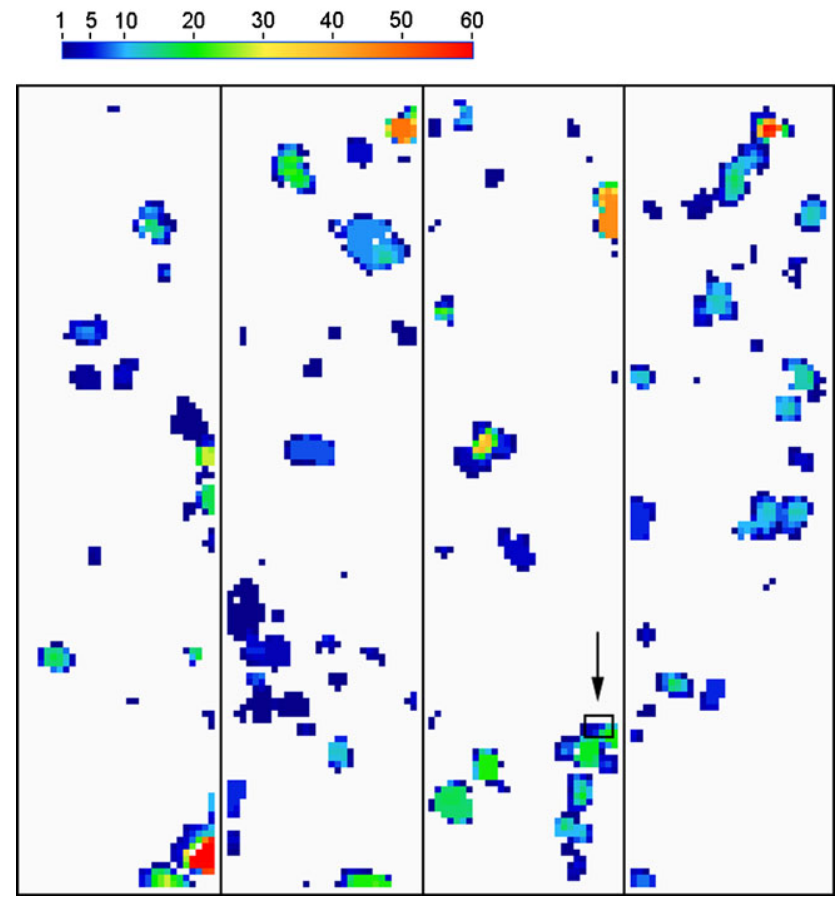

Fig. 6 Distribution of identified action potentials on $1 \mathrm{~mm}^{2}$ measured with Multi-Transistor Array. On each pixel, the number of detected action potentials is indicated in a color code. The data are separately collected at $24 \mathrm{kHz}$ with ten recordings for duration of $600 \mathrm{~ms}$ on each of four vertical stripes. Black rectangle marked by arrow: area of the chip that is chosen for Fig. 7

not be necessarily assigned to a single nerve cell that couples to a set of adjacent transistors. It may be due to several nerve cells that interact with the same set of adjacent transistors.

\subsection{Identification of firing neurons}

In order to determine the activity of a neuronal network, we must separate recordings of APs that belong to different cells. Two aspects must be considered: different transistors probe signals from one neuron and different cells couple to one sensor transistor. The situation is illustrated in Fig. 7 with recordings by 12 adjacent transistors that are marked in Fig. 6 by a black frame. Visual comparison indicates that AP recordings can be assigned to two different times and two different distributions in space. It suggests that the recordings belong to two different cells with an overlapping cellchip interaction.

Practically, the assignment of signals is achieved by an algorithm that evaluates the cross-correlation of the AP recordings for all pairs of spindle-shaped space-time volumes as described in the methods section. We consider only correlations in a subset of spindles where each spindle overlaps at least with one other spindle. A correlation matrix of 
such a set of spindles is shown in Fig. 8a. That matrix is sorted by an iterative algorithm with a result that is depicted in Fig. 8b. Visual inspection shows that the positive crosscorrelations belonging to two cells are localized in the lower left and upper right corners. By splitting the matrix at the boundary between these subpopulations the APs can be separated and assigned to two different cells.

Using the correlation method, 127 firing neurons can be identified in the map of APs of Fig. 7 and are marked in Fig. 9a as circles. Apparently, several of the clusters in Fig. 7 are partitioned into recordings from several adjacent neu-

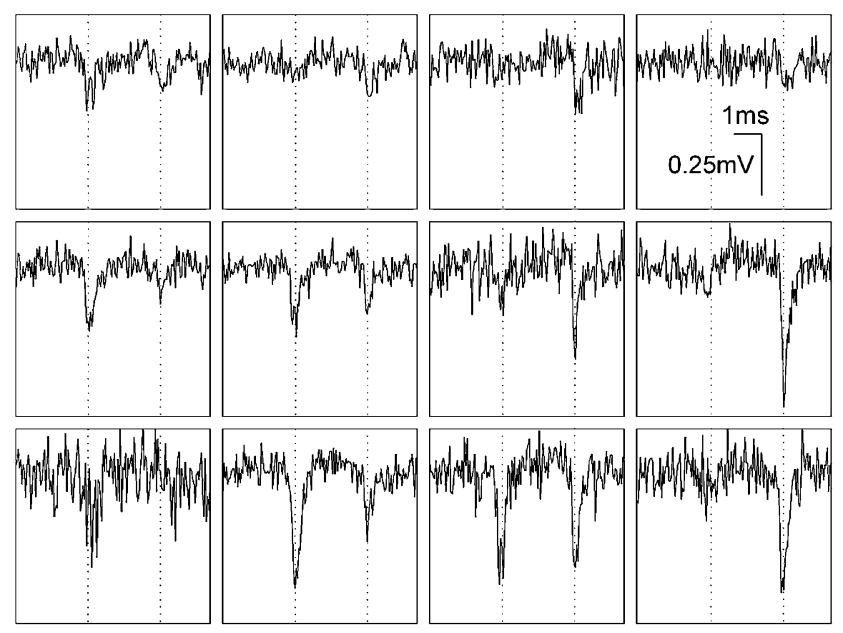

Fig. 7 Recordings of 12 sensor transistors (see black rectangle in Fig. 6). Dashed lines mark the time frames that refer to the recordings of two action potentials that belong to two different neurons (cells 105 and 106 in Fig. 9) rons. In Fig. 9b, the identified sites of firing neurons are superposed to a micrograph of the chip that shows blob-like features which indicate the position of neuronal cell bodies or clusters of cell bodies. A striking issue of Fig. $9 \mathrm{~b}$ is that the positions of recording often are not in coincidence with the position of such a blob. That mismatch confirms the assignment of the negative waveforms to the spike-generating part of the cells that is not at the cell body. Another important feature of Fig. 9b is that the number of firing neurons is far lower than the number of visible cell bodies. On the basis of the current MTA recordings, it is not possible to decide whether a large number of neurons do not participate in network activity or whether a large number of firing neurons are so weakly coupled to the chip that they cannot be identified.

\subsection{Network recording}

Multi-site recording with identified neurons is essential to investigate and understand the activity of neuronal networks. As an example, Fig. 10 shows the recordings of 23 neurons that were identified on one $250 \mu \mathrm{m} \times 1000 \mu \mathrm{m}$ strip during $600 \mathrm{~ms}$. Some APs can be seen by visual inspection. Others are identified by the correlation with neighboring data in time and space. All identified APs are marked by a red dash in the trace corresponding to the cell to which the AP was assigned.

The example in Fig. 10 reveals that there exists a spontaneous activity of the cultured network with bursts of duration of $600 \mathrm{~ms}$. The activity of neurons is correlated even when they are spatially separated for almost a millimeter.

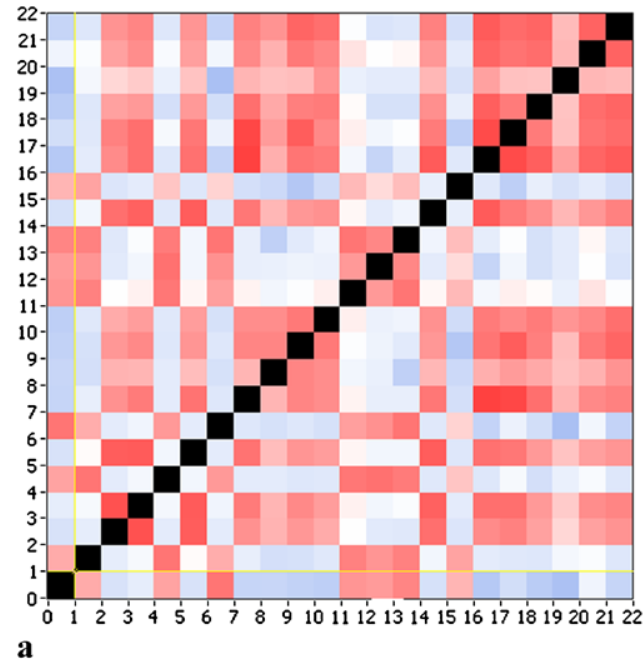

Fig. 8 Correlation matrix of all AP recordings (spindle-shaped volumes in space-time) from two neighboring neurons (cells a and b of Fig. 9). The value of the mutual correlation of pairs of spindles is colorcoded from 0 (blue) to 1 (red). For details of the calculation see Materials and Methods section. (a) Unsorted matrix. (b) Sorted matrix

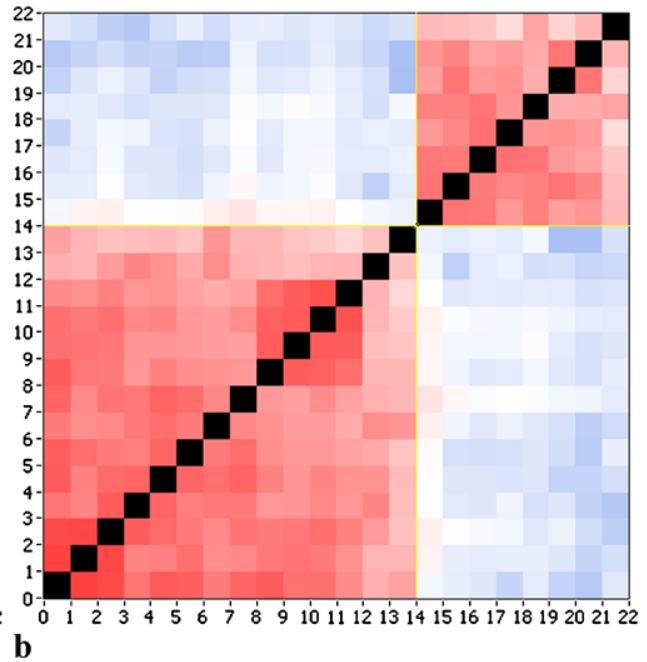

that shows how the matrix can be separated into two subpopulations of spindles that have high correlation values with spindles of the same subpopulation, but low correlation values with spindles of the other subpopulation. The two subpopulations of spindles represent two populations of action potentials belonging to two different cells 

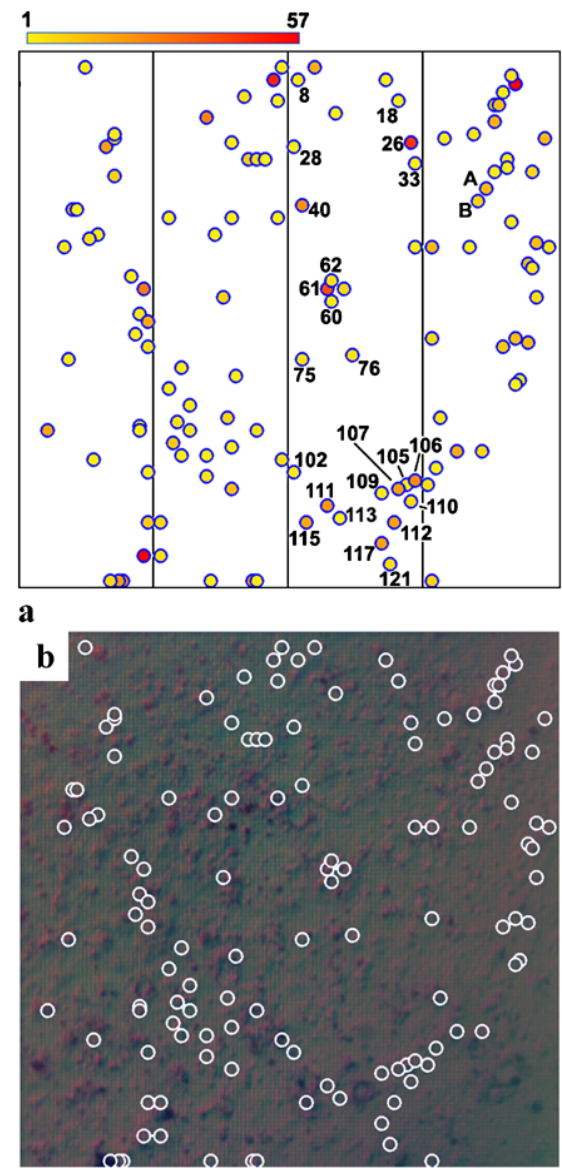

Fig. 9 Identified firing neurons on Multi-Transistor Array. (a) Positions of the 127 neurons with a color code that represents the activity. The numbers refer to the neurons in Fig. 10. (b) Superposition of the positions of electrically identified neurons and a micrograph of the chip. There is no clear relation of the position of the electrical activity (circles) and blob-like features that represent cell bodies or clusters of cell bodies

That correlation indicates that a single neuronal network is formed all over the chip. It must be emphasized that it is uncertain how many neurons contribute to the network. Many neurons in the culture may be silent in the interval of recording, many may participate in the activity but not interact with the chip.

\section{Conclusion}

In the present paper, the mapping of neuronal activity of cultured rat neurons is achieved on an area of $1 \mathrm{~mm}^{2}$ at a spatial resolution of $7.4 \mu \mathrm{m}$ and time resolution of $24 \mathrm{kHz}$ using a multi-transistor array made of $6.6 \mu \mathrm{m} \times 6.6 \mu \mathrm{m}$ sensors. Action potentials give rise to extracellular voltages with amplitudes in a range of $0.5 \mathrm{mV}$. Due to the high resolution in space and time and the application of correlation algorithms, single action potentials with amplitudes down to

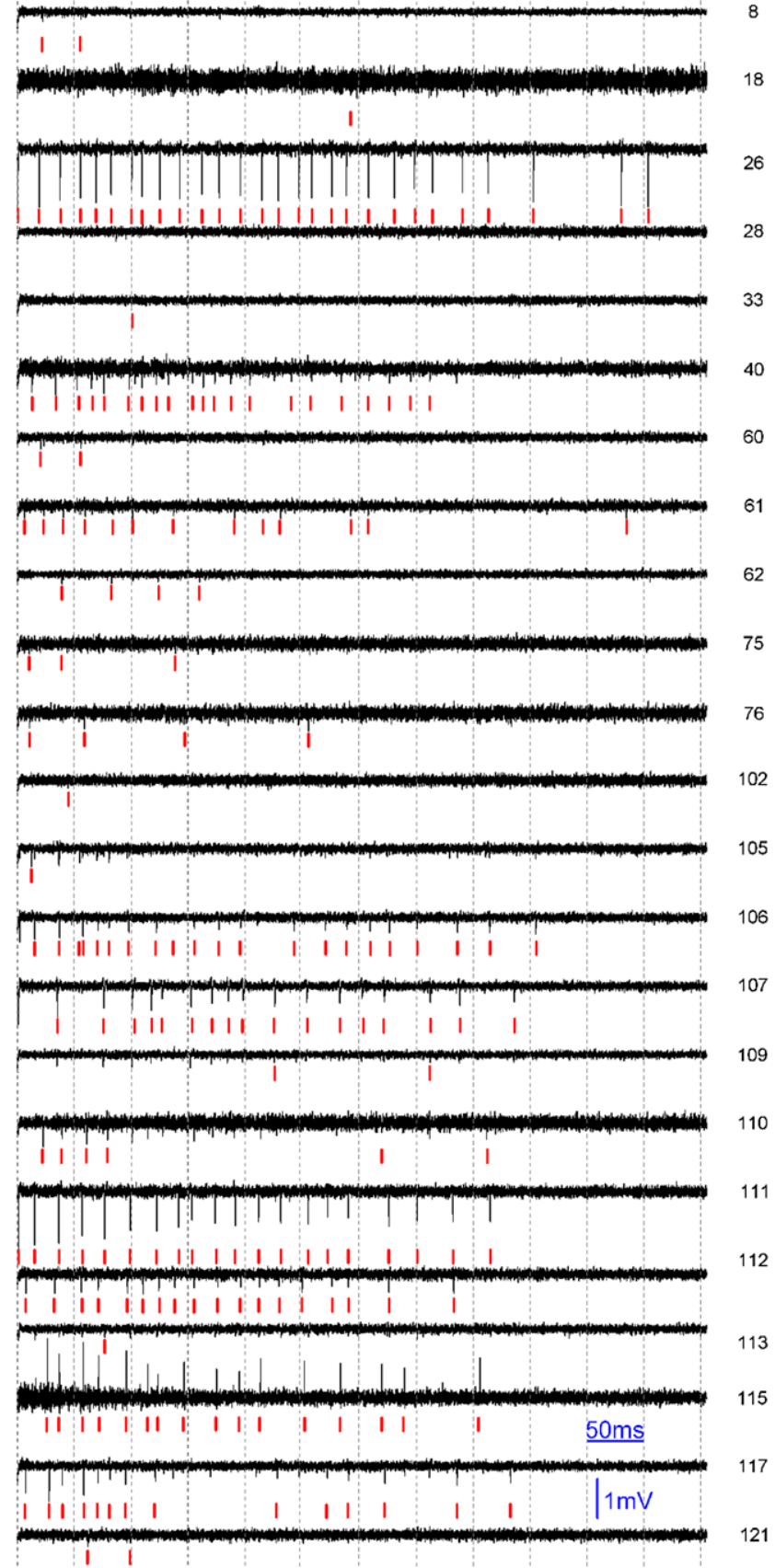

Fig. 10 Transistor recordings of 23 neurons during a burst of neuronal activity in a cultured network. The position of the neurons is shown in Fig. 9. Identified action potentials are marked by red dashes in those traces that correspond to the cells to which the action potential was assigned

$200 \mu \mathrm{V}$ could be identified and assigned to individual neurons in a dense network. This approach compensates a relatively high noise level inherent to the architecture of the chip that is optimized for highest density of sensors. As a consequence, a reliable detection of neuronal activity combined with complete spatiotemporal mapping at highest resolution is achieved. 
Acknowledgements We thank Doris Eckerlein for expert help with the cell culture, Alexander Kunze for support in amplifier electronics, and Reinhard Guckenberger for AFM measurements. The project was supported by the Max Planck Society and by an EU grant (NaChip project.)

Open Access This article is distributed under the terms of the Creative Commons Attribution Noncommercial License which permits any noncommercial use, distribution, and reproduction in any medium, provided the original author(s) and source are credited.

\section{References}

1. C.A. Thomas et al., Exp. Cell. Res. 74, 61 (1972)

2. G.W. Gross, E. Riedke, W. Kreutzberg, A. Meyer, Neurosci. Lett. 6, 101 (1977)

3. J. Novak, B. Wheeler, J. Neurosci. Methods 23, 149 (1988)

4. W.G. Regehr et al., J. Neurosci. Methods 30, 91 (1989)

5. W.L.C. Rutten, J. van Wier, J.H.M. Put, IEEE Trans. Bio. Med. Eng. 38, 192 (1991)

6. U. Egert et al., Brain Res. Protoc. 2, 229 (1998)

7. Y. Jimbo, H.P.C. Robinson, Bioelectrochemistry 51, 107 (2000)

8. P. Fromherz, A. Offenhausser, T. Vetter, J. Weis, Science 252, 1290 (1991)
9. P. Fromherz, C.O. Mueller, R. Weis, Phys. Rev. Lett. 71, 4079 (1993)

10. R. Schatzthauer, P. Fromherz, Eur. J. Neurosci. 10, 1956 (1998)

11. S. Vassanelli, P. Fromherz, Appl. Phys. A 66, 459 (1998)

12. M. Voelker, P. Fromherz, Small 1, 206 (2005)

13. M. Hutzler, P. Fromherz, Eur. J. Neurosci. 19, 2231 (2004)

14. F. Wallrapp, P. Fromherz, J. Appl. Phys. 99, 114103 (2006)

15. B. Eversmann et al., IEEE J. Solid State Circuits 38, 2306 (2003)

16. A. Lambacher et al., Appl. Phys. A 79, 1607 (2004)

17. M. Hutzler et al., J. Neurophysiol. 96, 1638 (2006)

18. F. Heer et al., IEEE J. Solid State Circuits 41, 1620 (2006)

19. U. Frey et al., Proceedings 2007 ISSCC, Session 8, p. 12 (2007)

20. K. Imfeld et al., IEEE Trans. Biomed. Bioeng. 55, 2064 (2008)

21. P. Fromherz, in Bioelectronics, ed. by I. Willner, E. Katz (Wiley, Weinheim, 2005), p. 339

22. P. Fromherz, Eur. Biophys. J. 28, 254 (1999)

23. M. Voelker, P. Fromherz, Phys. Rev. Lett. 96, 228102 (2006)

24. K. Goslin, H. Asmussen, G. Banker, in Culturing Nerve Cells, ed. by G. Banker, K. Goslin (MIT Press, Cambridge, 1998), p. 339

25. S. Rothman, W.M. Cowan, J. Comp. Neurol. 195, 141 (1981)

26. M. Meister, J. Pine, D.A. Baylor, J. Neurosci. Methods 51, 95 (1994)

27. A.M. Litten et al., IEEE Trans. Nucl. Sci. 51, 1434 (2004)

28. R. Segev et al., Nat. Neurosci. 7, 1155 (2004)

29. C. Stangl, P. Fromherz, Eur. J. Neurosci. 27, 958 (2008) 